\title{
Diagnosis of Cerebral Aneurysm Via Magnetic Resonance Angiography Screening: Emphasis on Legal Responsibility Increases False Positive Rate
}

\author{
Su-hee Cho, MD', Ji-ye Lee, MD³, Kyeong-hwa Ryu, MD", Dae Chul Suh, MD²
}

Purpose: False positive diagnoses of cerebral aneurysm via magnetic resonance angiography (MRA) screening may increase unnecessary cerebral catheter angiography. The purpose of this study was to investigate the effects of medical liability on medical decision-making during radiologic interpretation.

Materials and Methods: We included 56 consecutive patients who were referred with suspected aneurysm based on MRA or computed tomography angiography (CTA) and showed no aneurysm on subsequent digital subtraction angiography (DSA). MRA and CTA were reviewed twice by two neuroradiology fellows who were blind as to whether the suspected lesions were true aneurysms or not. The second review was repeated after proposing that their decision was subject to legal liability and they would be responsible for medico-legal problems related to their diagnoses. Diagnostic differences based on each review were analyzed, focusing on changes in false positive diagnosis rates.

Results: A total of 63 suspected aneurysmal lesions detected via MRA or CTA were found to be negative based on DSA. At first review, 32 lesions were diagnosed as true aneurysms by observer 1 and 27 by observer 2, corresponding to false positive rates of $51 \%$ and $43 \%$ respectively. At the second review, 39 lesions $(62 \%)$ were diagnosed by observer 1 , and $30(48 \%)$ by observer 2 . Thus, there was an overall increase in false positive aneurysm diagnosis of $11 \%$ for observer 1 and $5 \%$ for observer 2 , after emphasizing their responsibilities in the context of medical litigation.

Conclusion: Concerns about medical liability could result in increased false positive diagnoses of cerebral aneurysms via MRA screening. Whether repeated follow-up of the suspected lesion or catheter angiographic confirmation is better with regard to long-term patient outcomes requires further study.

Key Words : Intracranial aneurysm; Junctional dilatation; Magnetic resonance angiography;

Digital subtraction angiography

'Department of Neurosurgery, Gangneung Asan Medical Center, University of Ulsan College of Medicine, Seoul, Korea; ${ }^{2}$ Department of Radiology, Asan Medical Center, University of Ulsan College of Medicine, Seoul, Korea; ${ }^{3}$ Department of Radiology, Soonchunhyang University Bucheon Hospital, Gyeonggi-do, Korea; ${ }^{4}$ Deparment of Radiology, Gyeongsang National University School of Medicine and Gyeongsang National University Changwon Hospital, Changwon, Korea

Received: January 5, 2018; Revised: February 23, 2018; Accepted: February 25, 2018

Correspondence to: Dae Chul Suh, MD, PhD

Department of Radiology, Asan Medical Center, University of Ulsan College of Medicine, 88, Olympic-ro 43-gil, Songpa-gu, Seoul 05505, Korea Tel. +82.2.3010.4366 Fax.+82.2.476.0090 E-mail: dcsuh@amc.seoul.kr

This is an Open Access article distributed under the terms of the Creative Commons Attribution Non-Commercial License (http://creativecommons.org/licenses/by-nc/3.0) which permits unrestricted non-commercial use, distribution, and reproduction in any medium, provided the original work is properly cited. 
Medical error is unavoidable in clinical settings, despite the evolution of medical techniques and an accumulation of physicians' experiences and knowledge. Several studies have reported that radiologic practices are associated with medical error rates of approximately $3-5 \%$, as a day-to-day rate. ${ }^{1,2}$ Although most of these errors are minor and do not cause harm to patients, misdiagnoses are often implicated in medical litigation, which makes physicians feel guilty and they can also suffer economic and professional consequences. ${ }^{1,3}$ When clinicians interpret radiologic data, fear of medical litigation may affect their medical decisions. To avoid medical litigation, they may tend to interpret equivocal radiographic findings as abnormal lesions. This may result in increased false positive diagnoses.

We hypothesized that detection rates of cerebral aneurysms via magnetic resonance angiography (MRA) screening may increase after emphasizing the possibility of medical litigation. Therefore, the detection rate (i.e., false positive ratio) of aneurysm presence would be reflected in the findings of cerebral aneurysms in patients who underwent screening via cerebral MRA, especially in cases involving an inexperienced physician. Thus, the aim of the present study was to investigate such an effect in a blind manner in two neuroradiology fellows undergoing training, by evaluating associations between awareness of medical liability and false positive rates.

\section{MATERIALS AND METHODS}

The study was approved by the relevant institutional review board, and registered. Written informed consent from evaluated patients was not required because this study was conducted in a retrospective manner, and no additional imaging was performed as part of the study.

\section{Study population}

Fifty-six consecutive patients who had been proven not to be suffering from a definite aneurysm via digital subtraction angiography (DSA) were included in this study. All had been admitted to our neurointervention department for evaluation of a suspected intracranial aneurysm that was detected via MRA or computed tomography angiography (CTA). We performed DSA on these patients to confirm the diagnosis, after obtaining informed consent. When clarifying the presence of an aneurysm, an aneurysm was defined as a saccular outpouching lesion from a parent artery that had a clearly definable sac and neck in comparison with junctional dilatation, was triangular with regard to the shape of dilatation, and had a branching vessel from its apex..$^{4-6}$ In all included cases, three assessors agreed that the suspected lesion was non-aneurysmal on the basis of DSA data.

\section{Imaging modalities}

Initial MRA and CTA were performed via different protocols in each of the patients, because most of them had these procedures done at other institutions. However, all MRA included unenhanced 3D-TOF MRA images, and all CTA included contrast-enhanced $3 \mathrm{D}$ reconstructive images. All DSAs were performed at our institution via transfemoral catheterization. Conventional 2D-DSA was performed using a biplane angiographic system (Artis Zee biplane system; Siemens, Erlangen, Germany). For all patients, rotational angiography was performed with a 6-second $210^{\circ}$ rotational run and the injection of $3 \mathrm{~mL}$ of contrast medium per second. For suspected lesions, 3D images reconstructed by syngo Dyna3D (Siemens, Germany) were acquired and reviewed on site.

\section{Study design}

The aim of the study was to evaluate changes in false positive cerebral aneurysm diagnosis rates after emphasizing medical liability to inexperienced assessors. MRA or CTA results from all patients were reviewed twice by two neuroradiology fellows with 8 months experience in a neuroradiologic subspecialty. They were blinded to all clinical information and final results based on DSA, except the locations of the suspected aneurysmal lesions. They analyzed all 3DTOF MRA images and CTA images independently on an offline-workstation from multiple on-screen viewing angles. They could adjust appropriate thresholds of the window width and level to differentiate smaller lesions. The source images and maximum intensity projection images were not presented because they were not available for all cases. Diagnoses focused on the presence or absence of aneurysm were classified into one of the following four categories: 1 . Aneurysm; 2. Junctional dilatation; 3. Normal anatomy; 4. Others. "Others" included bulbous change, prominent origin of branch vessel, and flow artifact. One day after the first assessment, the second assessment was performed via the same method, but this time it was emphasized to the assessors that their diagnoses were subject to legal liability, and that they should be responsible for medico-legal problems related to their diagnoses. Thus, the assessors interpreted the same data they were given 
during the first review, with medical liability in mind. Based on these results, comparative analysis between the first and the second review was performed. To calculate false positive rates, true negative lesions were decided on the basis of angiographic results.

\section{RESULTS}

A total of 56 patients with 63 suspected aneurysmal lesions based on MRA or CTA were compared. The patient population consisted of 37 women $(66 \%)$ and 19 men (34\%), with a mean age of 51 years (range 2968 years). The imaging modalities utilized were CTA in 4 patients, and MRA in 52 patients. Thirty-seven patients underwent initial MRA or CTA at a community hospital and were referred to our institution for confirmatory diagnosis. Suspected aneurysms were located in the distal internal carotid artery $(n=39)$, middle cerebral artery $(n=9)$, anterior cerebral artery $(\mathrm{n}=8)$, and posterior circulation $(\mathrm{n}=7)$. The mean largest dimension of the suspected aneurysms was 2.3 $\mathrm{mm}$ (range, $1.5-8 \mathrm{~mm}$ ). On DSA, all of the suspected lesions included in the current study were revealed to be non-aneurysmal. The leading causes of false positives were junctional dilatation $(n=39)$, prominent origin of the branch $(n=6)$, fenestration $(n=4)$, bulbous change $(\mathrm{n}=5)$, artifact due to venous structure $(\mathrm{n}=4)$, arterial loop $(\mathrm{n}=3)$, and subtraction artifact ( $\mathrm{n}$ =2) (Fig. 1).

In the first review, observer 1 determined that there were $32(51 \%)$ aneurysms, $19(30 \%)$ junctional dilatations, and $9(14 \%)$ normal structures, and classified 3 (5\%) as "others" (Fig. 2). Observer 2 determined that there were 27 (43\%) aneurysms, 27 (43\%) junctional dilatations, and $6(10 \%)$ normal structures, and classified $3(5 \%)$ as others. False positive rates in the first review were $51 \%$ for observer 1 and $43 \%$ for observer 2. The second review resulted in differences in the diagnoses. In the second review, observer 1 diagnosed 39 (62\%) aneurysms, 17 (27\%) junctional dilatations, and $4(6 \%)$ normal structures, and classified $3(5 \%)$ as others. Observer 2 diagnosed 30 (48\%) aneurysms, 21 (33\%) junctional dilatations, and $6(10 \%)$ normal structures, and classified $6(10 \%)$ as others. The overall false positive rates in the second review were $62 \%$ for observer 1 and $48 \%$ for observer 2 . Therefore, there were overall increases in false positive aneurysm diagnoses of $11 \%$ for observer 1 and $5 \%$ for observer 2, after emphasizing the possibility of litigation arising from their diagnoses, and their potential responsibility for that litigation. Observer 1 shifted from a diagnosis of non-aneurysmal lesion to a diagnosis of aneurysm
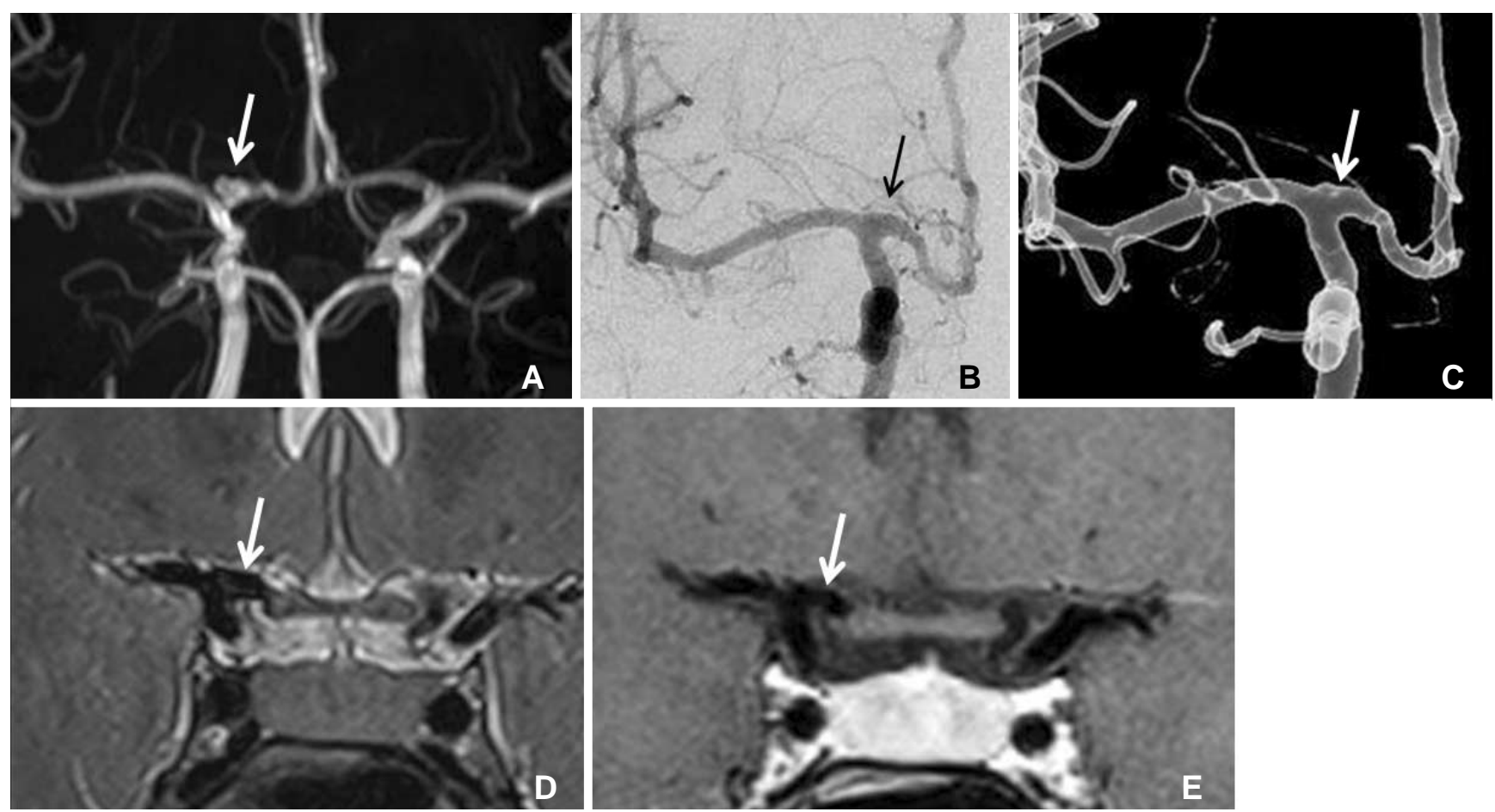

Fig. 1. False positive diagnosis of an aneurysm (arrows) in the right $A 1$. (A) An aneurysm was suggested in the right $A 1$ on $3 D$-TOF MRA. (B) There was no definite aneurysm on cerebral angiogram. (C) Three dimensional angiogram showed a mild dilatation of the proximal A1. There was neither abnormal vessel wall thickness (D) nor abnormal enhancement (E) on vessel wall MRI. 
A. Observer 1

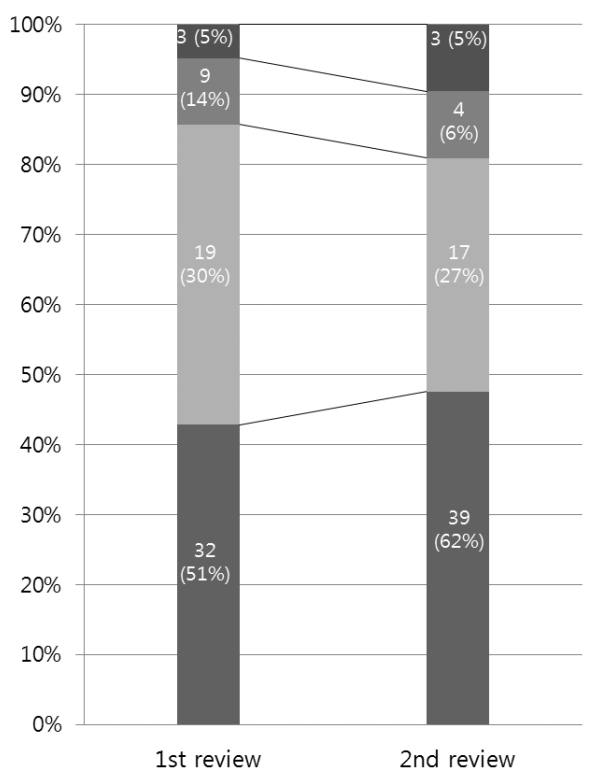

a others

normal

- junctional dilatation

- aneurysm

A

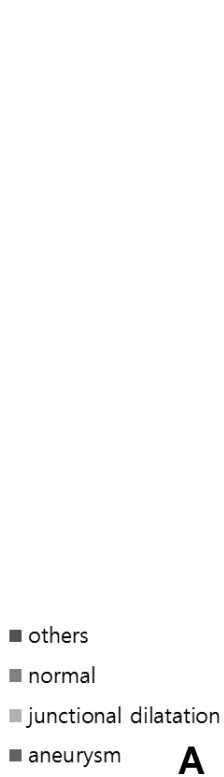

B. Observer 2

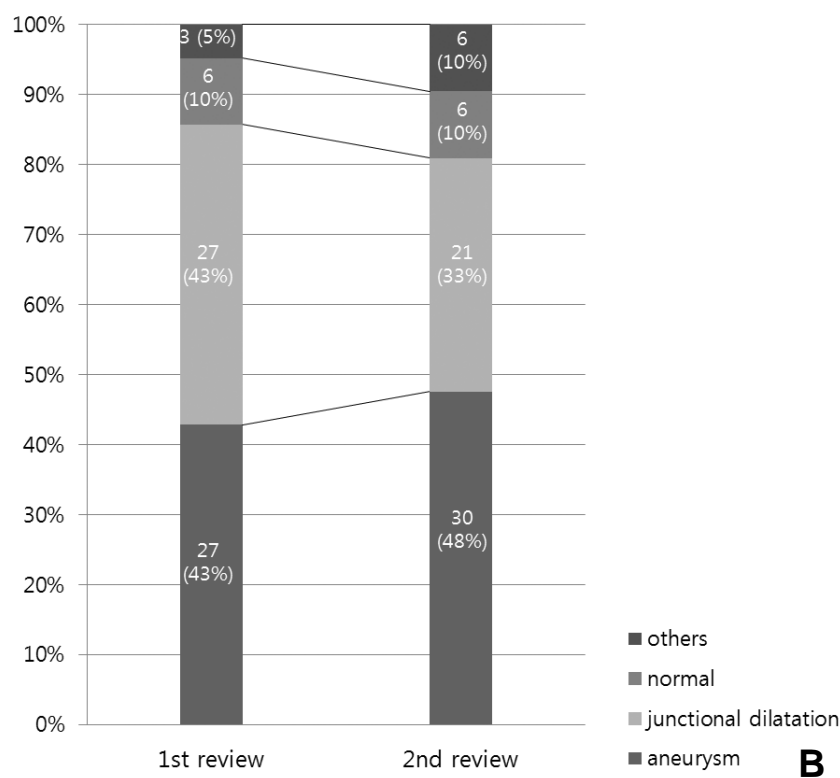

Fig. 2. Changes in diagnostic results from observer 1 (A) and observer 2 (B). Note increased false positive diagnosis rates of aneurysm after emphasizing the possibility of medical litigation (2nd review) in both observers

for $11(18 \%)$ lesions, and observer 2 shifted from a diagnosis of non-aneurysmal lesion to a diagnosis of aneurysm for $8(13 \%)$ lesions. Observer 1 shifted from a diagnosis of aneurysmal lesion to a diagnosis of nonaneurysmal lesion in $4(6 \%)$ cases, and observer 2 made such a shift in $5(8 \%)$ cases. Diagnostic results remained unchanged for $46(73 \%)$ lesions for observer 1, and 47 (75\%) lesions for observer 2.

\section{DISCUSSION}

False positive diagnosis is a test result that indicates that a person has a specific disease or condition when the person actually does not have that disease or condition. False positive rates depend on characteristics of the patient, the tests involved, and the assessors. ${ }^{7}$ It has been reported that physicians' anxiety about misdiagnosis and fear of resulting litigation may be particularly likely to give rise to false positive diagnoses. ${ }^{8}$ This has become a more important issue recently, as radiologists are now exposed to risks of medical litigation more often. Furthermore, medical liability can be a matter of great concern in high-risk specialties such as neuroradiology, in which misdiagnoses are potentially associated with extremely adverse outcomes. ${ }^{9}$

The current study revealed considerable changes in radiologic interpretation after notification of legal responsibility. The shifts from diagnoses of nonaneurysmal lesions (junctional dilatation, or normal, or "others") to diagnoses of aneurysms were remarkable, and resulted in increases in false positive rates from $51 \%$ to $62 \%$ and $43 \%$ to $48 \%$ in the two relatively inexperienced assessors. Such inconsistencies in diagnoses may be affected by various factors including insufficient knowledge, limited experience and poor quality of image data. Considering all the conditions except attention for medical liability was given for both two reviews, fear of medical litigation may be a major cause of increased false positive rates. Several studies investigating medical liability and defensive medicine have yielded comparable results. ${ }^{10,11}$ In one survey, $71.3 \%$ of neurosurgeons confirmed that medical liability affected their decisions, and $72 \%$ of them ordered imaging studies for defensive purposes. ${ }^{9}$ Studdert et al. ${ }^{12}$ reported that $59 \%$ of surgeons ordered more tests than were medically indicated, and 32\% suggested invasive procedures to confirm diagnosis because of liability concerns. This trend led to higher healthcare expenditure and the performance of unnecessary medical procedures on patients who would be anxious about their medical conditions. ${ }^{7,10}$

Concern over the $0.05-1.00 \%$ annual rupture risk of unruptured aneurysms and subsequent disability may contribute to healthy aged people getting screening tests even though most unruptured intracranial 


\section{Su-hee Cho, et al.}

aneurysms are asymptomatic. ${ }^{13}$ For aneurysm screening, MRA or CTA are generally used. ${ }^{14-16}$ Based on the results of these examinations, DSA would be performed as the gold standard to confirm the final diagnosis, once the suspicion of aneurysm was mentioned on a radiologist's report. ${ }^{14,17}$ While the omission of a diagnosis despite a suspicion can result in serious medical conditions or legal consequences, aneurysm screening is highly sensitive, giving rise to increased false positive diagnoses. Several studies have reported false positive rates associated with aneurysm screening. Okuyama et al. ${ }^{18}$ reported that 3 of 66 (4.5\%) aneurysms detected by MRA or CTA were false positives. A meta-analysis performed by Hiratsuka et al. ${ }^{19}$ revealed that the overall specificity for the diagnosis of aneurysm via MRA ranged from $50 \%$ to $100 \%$, which is relatively comparable to our results ( $43 \%$ to $62 \%)$. He concluded that the need for high sensitivity in order to be able to rule out the presence of aneurysm results in higher false positive rates. While sensitivity is emphasized, specificity also has to be excellent, because one does not want to perform confirmatory DSA unnecessarily. ${ }^{20}$ Although an accurate diagnosis could be achieved via catheter angiography, DSA is associated with a $0.1-0.5 \%$ risk of persistent neurological deficits, and a $1-4 \%$ risk of complications related to arterial puncture and catheter manipulation. . $^{13,17,18,21}$

Should we routinely perform DSA whenever a patient with suspected aneurysm based on initial screening is referred from a community hospital? Despite the fact that many studies have established that MRA or CTA with advanced imaging techniques can achieve accurate diagnosis of intracranial aneurysms with high sensitivity and specificity rates, there are several factors that lead to the performance of DSA. ${ }^{15-17,19,22,23}$ These include the fact that missed aneurysm is potentially life threatening, the patient's need for reassurance, and the physician's fear of misdiagnosis causing medical and legal problems. These factors may affect medical decisions differently, depending on the social environment. In Korea, according to guidelines for intracranial aneurysms suggested by the Korean Society of Interventional Neuroradiology, screening for intracranial aneurysm is recommended in patients with highlevel risk factors such as a family history of intracranial aneurysm, an associated medical condition, or a history of previous aneurysmal rupture. ${ }^{24}$ The current study included 37 patients who had no aneurysm risk factors and no specific neurologic symptoms, and underwent brain MRA or CTA during the course of a medical check-up at a community hospital. When they visited our institution, they had already received a warning about aneurysm, and had been informed that it could be fatal and that treatment may be required. Most of them were extremely anxious, and expressed a strong desire to obtain an accurate diagnosis. Thus, the clinicians may have been faced with a dilemma with regard to whether to perform DSA or not, particularly in cases where the possibility of aneurysm was regarded as low. When legal aspects were considered, they became especially likely to perform DSA. Therefore, the final judgments made by the physician and the patient must be based on multiple factors including medical, social, and personal considerations.

The present study has several limitations. First, false negative rates could not be investigated because the study only included patients who were ultimately found to be aneurysm negative via DSA. Further study including true aneurysm as well as false positive lesion could make more meaningful results. Second, the assessors were neuroradiology fellows in training who had limited experience in their neuroradiologic subspecialty. Thus, their attitudes and levels of concentration during their assessments may have differed from those that are typical in clinical practice. These factors may also have contributed to the interobserver difference. Third, heterogeneity of given imaging data due to different imaging protocols and differences in image quality associated with screening performed in a primary health center can affect misdiagnoses. Fourth, intraobserver variation did not considered in this study. To correct this variation, several times of interpretation with enough time interval should be performed in further study. Fifth, various factors may be related to increased false positive rates, for example expectation bias. The assessor may expect a positive diagnosis because the presence of suspected aneurysmal lesion has already been proposed before their assessment. Concern about assessment discrepancy between colleagues may also affect their decisions. To generate more objective information about correlations between concerns about liability and false positive diagnoses, multivariate analysis with more experienced radiologists and a large sample size needs to be performed.

\section{CONCLUSION}

Concerns about medical liability could affect physicians' medical decisions. This study revealed that an emphasis on liability resulted in increased false positive rates with regard to diagnoses of intracranial aneurysm based on MRA and CTA screening. 


\section{False Positive Diagnosis of Cerebral Aneurysm}

\section{References}

1. Pinto A, Brunese L, Pinto F, Reali R, Daniele S, Romano L. The concept of error and malpractice in radiology. Semin Ultrasound CT MR 2012;33:275-279

2. Brady AP. Error and discrepancy in radiology: Inevitable or avoidable? Insights Imaging 2016

3. Fileni A, Magnavita N, Mirk P, Iavicoli I, Magnavita G, Bergamaschi A. Radiologic malpractice litigation risk in italy: An observational study over a 14-year period. AJR Am J Roentgenol 2010;194:1040-1046

4. Villablanca JP, Jahan R, Hooshi P, Lim S, Duckwiler G, Patel A, et al. Detection and characterization of very small cerebral aneurysms by using $2 \mathrm{~d}$ and $3 \mathrm{~d}$ helical ct angiography. AJNR Am J Neuroradiol 2002;23:1187-1198

5. Fischer S, Hopf N, Henkes H. Evolution from an infundibulum of the posterior communicating artery to a saccular aneurysm. Clin Neuroradiol 2011;21:87-90

6. Satoh T, Omi M, Ohsako C, Fujiwara K, Tsuno K, Sasahara W, et al. Differential diagnosis of the infundibular dilation and aneurysm of internal carotid artery: Assessment with fusion imaging of $3 \mathrm{~d} \mathrm{mr}$ cisternography/angiography. AJNR Am J Neuroradiol 2006;27:306-312

7. Germino JC, Elmore JG, Carlos RC, Lee CI. Imaging-based screening: Maximizing benefits and minimizing harms. Clinical imaging 2016;40:339-343

8. Lee CS, Nagy PG, Weaver SJ, Newman-Toker DE. Cognitive and system factors contributing to diagnostic errors in radiology. AJR Am J Roentgenol 2013;201:611-617

9. Nahed BV, Babu MA, Smith TR, Heary RF. Malpractice liability and defensive medicine: A national survey of neurosurgeons. PLoS One 2012;7:e39237

10. Magnavita N, Fileni A, Mirk P, Magnavita G, Ricci S, Cotroneo AR. Malpractice claims in interventional radiology: Frequency, characteristics and protective measures. Radiol Med 2013;118: 504-517

11. Mavroforou A, Giannoukas A, Mavrophoros D, Michalodimitrakis E. Physicians' liability in interventional radiology and endovascular therapy. Eur J Radiol 2003;46:240-243

12. Studdert DM, Mello MM, Sage WM, DesRoches CM, Peugh J, Zapert K, et al. Defensive medicine among high-risk specialist physicians in a volatile malpractice environment. JAMA 2005;293:2609-2617

13. Keedy A. An overview of intracranial aneurysms. Mcgill J Med
2006;9:141-146

14. Adams WM, Laitt RD, Jackson A. The role of mr angiography in the pretreatment assessment of intracranial aneurysms: A comparative study. AJNR Am J Neuroradiol 2000;21:1618-1628

15. Hoh BL, Cheung AC, Rabinov JD, Pryor JC, Carter BS, Ogilvy CS. Results of a prospective protocol of computed tomographic angiography in place of catheter angiography as the only diagnostic and pretreatment planning study for cerebral aneurysms by a combined neurovascular team. Neurosurgery 2004;54:1329-1340; discussion 1340-1322

16. Mallouhi A, Felber S, Chemelli A, Dessl A, Auer A, Schocke M, et al. Detection and characterization of intracranial aneurysms with $\mathrm{mr}$ angiography: Comparison of volume-rendering and maximum-intensity-projection algorithms. AJR Am J Roentgenol 2003; 180:55-64

17. Sailer AM, Wagemans BA, Nelemans PJ, de Graaf R, van Zwam WH. Diagnosing intracranial aneurysms with mr angiography: Systematic review and meta-analysis. Stroke 2014;45:119-126

18. Okuyama T, Saito K, Hirano A, Takahashi A, Hashimoto Y, Inagaki T. Diagnosis of unruptured cerebral aneurysms using magnetic resonance angiography and three dimensional computed tomographic angiography. No Shinkei Geka 1997;25:1073-1079

19. Hiratsuka Y, Miki H, Kiriyama I, Kikuchi K, Takahashi S, Matsubara I, et al. Diagnosis of unruptured intracranial aneurysms: 3t mr angiography versus 64-channel multi-detector row ct angiography. Magn Reson Med Sci 2008;7:169-178

20. Wardlaw JM, White PM. The detection and management of unruptured intracranial aneurysms. Brain 2000;123 (Pt 2):205-221

21. Kaufmann TJ, Huston J 3rd, Mandrekar JN, Schleck CD, Thielen KR, Kallmes DF. Complications of diagnostic cerebral angiography: Evaluation of 19,826 consecutive patients. Radiology 2007; 243:812-819

22. McKinney AM, Palmer CS, Truwit CL, Karagulle A, Teksam M. Detection of aneurysms by 64-section multidetector ct angiography in patients acutely suspected of having an intracranial aneurysm and comparison with digital subtraction and $3 \mathrm{~d}$ rotational angiography. AJNR Am J Neuroradiol 2008;29:594-602

23. van Gelder JM. Computed tomographic angiography for detecting cerebral aneurysms: Implications of aneurysm size distribution for the sensitivity, specificity, and likelihood ratios. Neurosurgery 2003;53:597-605; discussion 605-596

24. Jeong HW, Seo JH, Kim ST, Jung CK, Suh SI. Clinical practice guideline for the management of intracranial aneurysms. Neurointervention 2014;9:63-71 